\title{
Assessment of the praxis circuit in glioma surgery to reduce the incidence of postoperative and long-term apraxia: a new intraoperative test
}

\author{
Marco Rossi, MD,, Luca Fornia, PhD, ${ }^{3}$ Guglielmo Puglisi, PhD, ${ }^{2,3}$ Antonella Leonetti, ${ }^{2,3}$ \\ Gianmarco Zuccon, MD, ${ }^{1}$ Enrica Fava, MD, PhD, ${ }^{2}$ Daniela Milani, ${ }^{2}$ Alessandra Casarotti, PhD, ${ }^{1,2}$ \\ Marco Riva, MD, ${ }^{1,2}$ Federico Pessina, MD, ${ }^{2}$ Gabriella Cerri, MD, PhD, ${ }^{3}$ and Lorenzo Bello, MD ${ }^{1,2}$ \\ ${ }^{1}$ Unit of Neurosurgical Oncology, Department of Hematology and Hemato-Oncology, and 'Laboratory of Motor Control, \\ Department of Medical Biotechnology and Translational Medicine, Università degli Studi di Milano; and 2Unit of Neurosurgical \\ Oncology, Humanitas Research Hospital, Rozzano, Italy
}

OBJECTIVE Apraxia is a cognitive-motor deficit affecting the execution of skilled movements, termed praxis gestures, in the absence of primary sensory or motor disorders. In patients affected by stroke, apraxia is associated with lesions of the lateral parietofrontal stream, connecting the posterior parietal areas with the ventrolateral premotor area and subserving sensory-motor integration for the hand movements. In the neurosurgical literature to date, there are few reports regarding the incidence of apraxia after glioma surgery. A retrospective analysis of patients who harbored a glioma around the central sulcus and close to the parietofrontal circuits in depth showed a high incidence of long-term postoperative hand apraxia, impairing the patients' quality of life. To avoid the occurrence of postoperative apraxia, the authors sought to develop an innovative intraoperative hand manipulation task (HMt) that can be used in association with the brain mapping technique to identify and preserve the cortical and subcortical structures belonging to the praxis network. METHODS The intraoperative efficacy of the HMt was investigated by comparing the incidence of postoperative ideomotor apraxia between patients undergoing mapping with $(n=79)$ and without $(n=41)$ the HMt. Patient groups were balanced for all demographic and clinical features.

RESULTS In patients with lesions in the dominant hemisphere, the HMt dramatically reduced the incidence of apraxia, with a higher sensitivity for the ideomotor than for the constructional abilities; patients with lesions in the nondominant hemisphere benefitted from the HMt for both ideomotor and constructional abilities. The administration of the test did not reduce the extent of resection.

CONCLUSIONS The HMt is a safe and feasible intraoperative tool that allowed surgeons to prevent the occurrence of long-term hand apraxia while attaining resection goals for the surgical treatment of glioma.

https://thejns.org/doi/abs/10.3171/2017.7.JNS17357

KEY WORDS intraoperative brain mapping; motor control; neurophysiology; neuropsychology; oncology

$\mathrm{P}$ RAXIS, the ability to perform complex motor gestures, is essential for the proper use of tools and objects in the environment. The loss of praxis gestures in the absence of primary sensory (deafness, blindness, or visual and tactile agnosia) or motor (palsy, tremor, ataxia, or hypokinesia and/or hyperkinesia) disorders ${ }^{16}$ is defined as apraxia. Apraxia is a cognitive-motor disorder and is clinically classified based on the body segments affected (limb or orofacial apraxia) and on the specific cognitive impairment (ideomotor or ideative). Here we specifically focused on ideomotor apraxia, a disturbance in timing, sequencing, and spatial organization of movements that causes an inability to correctly imitate gestures and mimic tool use.

The disclosure of the specific lesions correlating with apraxia may shed light on the neural network underlying praxis. So far, apraxia has been investigated with neuroimaging or qualitative behavioral studies,${ }^{19}$ suggesting the

ABBREVIATIONS AG = angular gyrus; DES = direct electrical stimulation; $E M G$ = electromyographic; $E O R=$ extent of resection; $H M t=$ hand manipulation task; $M 1$ = primary motor cortex; S1 = primary somatosensory cortex; SMG = supramarginal gyrus; vIPM = ventrolateral premotor cortex.

SUBMITTED February 10, 2017. ACCEPTED July 6, 2017.

INCLUDE WHEN CITING Published online February 23, 2018; DOI: 10.3171/2017.7.JNS17357. 

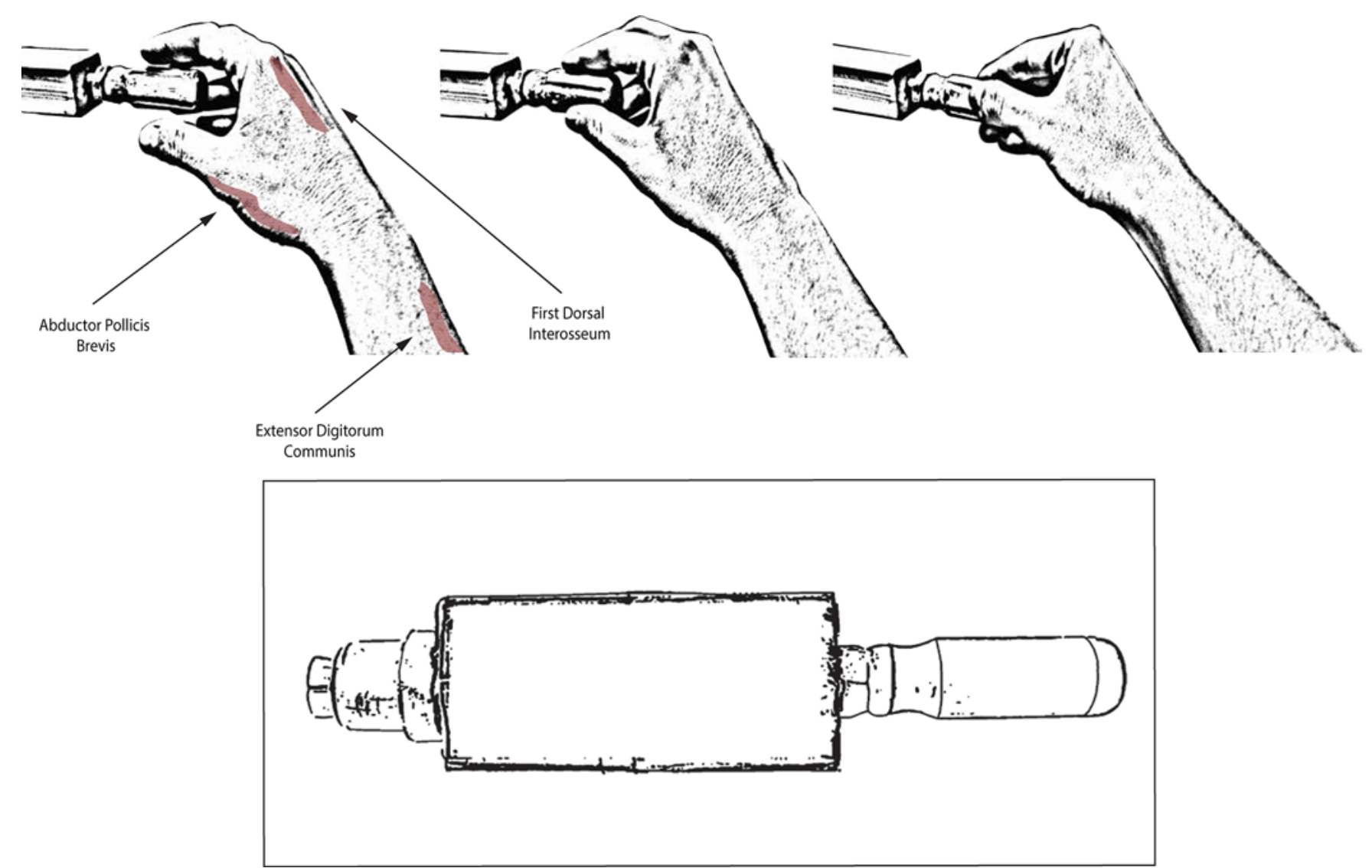

FIG. 1. Upper: The 3 drawings summarize the steps of the movement necessary to properly perform the task and the muscles used to execute it, for its proper evaluation. Lower: A sketch of the view from the top of the tool. Copyright Luca Fornia. Published with permission. Figure is available in color online only.

involvement of a parietofrontal network ${ }^{4,14,18,22}$ connecting the supramarginal gyrus (SMG) and angular gyrus (AG) to the ventrolateral premotor cortex (vlPM). In monkeys and humans, this circuit was recently defined as the "lateral grasping network," and demonstrated to be specifically active during hand movement in light and in darkness, pointing to its crucial role in hand tool manipulation even in a haptically driven context. ${ }^{3,4,10,22}$ In humans, this network is also defined as the praxis representation network and serves as the foundation for praxis abilities. These include more elementary hand movements, such as prehension requiring different types of grips based on physical features of objects, and integration and transformation of conceptual and sensorimotor information into purposeful skilled acts (praxis), such as processing and/or retrieval and control of tool-use pantomimes and other symbolic gestures. ${ }^{13}$

Apraxia is a relevant concern in brain tumor surgery. In fact, a retrospective analysis of patients with glioma at depths to approximately $2 \mathrm{~cm}$ from the precentral and postcentral sulcus demonstrated that upper-limb apraxia is a frequent postoperative deficit with a negative longterm impact on patient quality of life., ${ }^{917}$ Despite the intraoperative neurophysiological mapping routinely used in brain tumor resection, which allows us to preserve the motor functions (i.e., the general ability to move arms and/or hands), difficulties in programming and execution of complex action or fine movements are often reported postoperatively by patients. This evidence raised the need to optimize the current brain mapping protocols by incorporating new and more ecological intraoperative tasks designed to identify the praxis networks and reduce the occurrence of postsurgical hand apraxia, while maintaining the main functional and oncological goals. For these reasons, we designed the hand manipulation task (HMt), which requires hand-object interaction and the correct execution of fine finger movement (see Methods and Fig. 1). During intraoperative mapping, direct electrical stimulation (DES) delivered at the cortical and subcortical level while the patient was performing the HMt was expected to impair the performance of the task only when applied on areas and/or fibers involved in the neural circuit controlling praxis gestures. These are the eloquent praxis-related cortical or subcortical sites that have to be preserved to reduce the occurrence of postoperative permanent apraxia. The impact of the HMt was evaluated as follows: 1) occurrence of postoperative ideomotor apraxia, 2) specificity of the HMt (determined by comparing postoperative apraxia deficit and constructional abilities), 3) feasibility and reproducibility during surgery, and 4) achievement of oncological goal measured as extent of resection (EOR).

The characterization of the specific behavioral and muscle-related outcomes induced by DES during task performance will provide a further guide for resection. 
TABLE 1. Demographic and clinical variables for patients in groups $A$ and $B$

\begin{tabular}{|c|c|c|}
\hline \multirow[b]{2}{*}{ Variable } & \multicolumn{2}{|c|}{ No. $(\%)$} \\
\hline & Group A & Group B \\
\hline \multicolumn{3}{|l|}{ Laterality } \\
\hline $\mathrm{Rt}$ & $25(32)$ & $24(59)$ \\
\hline $\mathrm{Lt}$ & $54(68)$ & $17(41)$ \\
\hline \multicolumn{3}{|l|}{ Location } \\
\hline Parietal & $27(34)$ & $18(44)$ \\
\hline Frontal & $52(66)$ & $23(56)$ \\
\hline \multicolumn{3}{|l|}{ Relapse } \\
\hline Yes & $25(32)$ & $17(41)$ \\
\hline No & $54(68)$ & $24(59)$ \\
\hline \multicolumn{3}{|l|}{ WHO grade } \\
\hline I & $0(0)$ & $1(2)$ \\
\hline II & $41(52)$ & $22(54)$ \\
\hline III & $28(35)$ & $10(24)$ \\
\hline IV & $10(13)$ & $8(20)$ \\
\hline \multicolumn{3}{|l|}{ Histology } \\
\hline Oligodendroglioma & $17(21)$ & $9(22)$ \\
\hline Astrocytoma & $44(56)$ & $22(54)$ \\
\hline Glioblastoma & $10(13)$ & $8(19)$ \\
\hline Other & $8(10)$ & $2(5)$ \\
\hline \multicolumn{3}{|l|}{ Sex } \\
\hline Male & $45(57)$ & $24(59)$ \\
\hline Female & $34(43)$ & $17(41)$ \\
\hline \multicolumn{3}{|l|}{ Handedness } \\
\hline Rt & $75(95)$ & $38(93)$ \\
\hline Lt & $4(5)$ & $3(7)$ \\
\hline
\end{tabular}

\section{Methods}

\section{Patient Population}

We enrolled 120 patients treated at Humanitas Research Hospital between January 2013 and October 2016. All patients fulfilled both of the following criteria: 1) presence of a glial neoplasm located within $2 \mathrm{~cm}$ of the precentral and postcentral sulcus, and 2) absence of hand motor, praxis, somatosensory, or visual deficits on admission.

Patients were divided into 2 groups: A (the prospective study group) and B (the retrospective control group). Group A included patients who underwent surgery with the aid of brain mapping performed with the HMt, whereas group B included patients who underwent surgical procedures without the HMt. Group A patients $(n=79)$ were enrolled between August 2014 and October 2016. These were patients in whom we performed standard motor mapping with the HMt. Group B patients $(n=41)$ were enrolled between January 2013 and July 2014. These were patients in whom we performed standard motor mapping only.

Language or visual mapping was also performed when required by the clinical context. Demographic and clinical features of the 2 groups are reported in Table 1. Once the 2 groups were defined, their comparability on the main clinical variables was verified. Given that patients in group B were selected retrospectively from those fulfilling the criteria, the total number of patients that resulted was different in the 2 groups. However, this discrepancy has been overcome by the observation that groups A and B matched with regard to the main clinical variables: sex, laterality, tumor location, relapse, grade, and histology, but not the tumor volume. A chi-square test was performed to assess the comparability of the 2 groups. Notably, in both groups resection was performed according to functional boundaries, independently from the tumor volume.

To evaluate its efficacy in the largest population possible, the HMt was applied to a general population of patients, without any initial selection. All patients gave informed consent. Patients showing postoperative motor or sensory deficits were excluded from the analysis, which was mainly focused on apraxia outcome.

\section{Radiological Evaluation}

Preoperative MRI was performed on a Philips-Intera 3-T unit and acquired for lesion morphological and volumetric assessment. The MR protocol included the following: 1) axial 3D FLAIR, 2) post-Gd 3D T1-weighted fast field echo, and 3) diffusion-weighted imaging and apparent diffusion coefficient diffusion-weighted imaging.

Volumetric analysis was used to estimate tumor volume. The mean lesion volume was $26.83 \pm 19.93 \mathrm{~cm}^{3}$ (range 3.28-97.98 $\mathrm{cm}^{3}$ ) in group A and $44.91 \pm 31.54$ $\mathrm{cm}^{3}$ (range 3.84-105.6 $\mathrm{cm}^{3}$ ) in group B. Lesion volume was computed onto FLAIR volumetric sequences with manual segmentation performed using iPlan Cranial software (Brainlab AG) by 2 investigators (Rossi and Riva). The FLAIR hyperintense or T1-weighted, Gd-enhanced signal abnormalities were included in the lesion load for low-grade or high-grade gliomas, respectively, and were reported in cubic centimeters. Patients underwent both an immediate (within 48 hours) and a 3-month postoperative MR scan (volumetric FLAIR and post-Gd T1-weighted images) to estimate the EOR. The EOR corresponded to the percentage of the volume resected with respect to the preoperative volume: (preoperative volume - postoperative volume)/preoperative volume. Postoperative diffusionweighted MR scans were also performed to check for ischemic damage.

\section{General Neuropsychological Evaluation}

All patients underwent an extensive neuropsychological evaluation before and immediately after surgery (at 5-7 days), and within 1-3 months of surgery. The administered battery included tests assessing general intelligence, language, and executive functions as well as memory, constructional, and attentional abilities (Table 2).

\section{Upper-Limb Praxis Assessment}

Upper-limb apraxia was evaluated in both groups by using the "ideomotor-apraxia test," 25 based on the imitation of hand gestures; this test is considered the gold standard for detecting apraxia. During the test, patients were asked to imitate a variety (precisely 10 items) of intransitive gestures (not requiring the use of objects) pre- 
TABLE 2. General neuropsychological evaluation

\begin{tabular}{|c|c|}
\hline $\begin{array}{l}\text { Cognitive } \\
\text { Functions }\end{array}$ & Test \\
\hline Language & $\begin{array}{l}\text { Token Test (De Renzi \& Faglioni) } \\
\text { Picture naming of objects \& actions (Crepaldi et al.) } \\
\text { Verbal fluency on phonemic \& semantic cue (Novelli } \\
\text { et al.) }\end{array}$ \\
\hline \multicolumn{2}{|l|}{ Memory } \\
\hline \multirow[t]{2}{*}{ Short term } & Digit span forward \& backward (Monaco et al.) \\
\hline & Corsi span forward \& backward (Carlesimo et al.) \\
\hline \multirow[t]{2}{*}{ Long term } & Word list learning (Carlesimo et al.) \\
\hline & $\begin{array}{l}\text { Reproduction of the Rey-Osterrieth complex figure } \\
\left(\text { Caffarra et al. }{ }^{5}\right)\end{array}$ \\
\hline $\begin{array}{l}\text { Executive } \\
\text { functions }\end{array}$ & $\begin{array}{l}\text { Raven's Coloured Progressive Matrices (Basso et al.) } \\
\text { Weigl Color Form Sorting Test (Laiacona et al.) }\end{array}$ \\
\hline Attention & $\begin{array}{l}\text { Stroop Color and Word Test (Caffarra et al. }{ }^{6} \text { ) } \\
\text { Trail Making Test (Giovagnoli et al.) }\end{array}$ \\
\hline
\end{tabular}

sented by the neuropsychologist; patients were instructed to use both the arm ipsilateral and that contralateral to the affected hemisphere. No verbal description of the movements to be imitated was suggested. When an item was not reproduced correctly on the first demonstration, a second demonstration was given. Each item that was performed flawlessly on the first or second demonstration was scored 2 or 1 , respectively; in case of unsatisfactory reproduction, the item was scored 0 .

\section{Surgical Procedure}

Resection was accomplished according to functional boundaries (motor, language, visual, and cognitive). A craniotomy was performed to expose the tumor area and a limited amount of surrounding tissue. Surgery was performed in all patients under asleep-awake-asleep anesthesia, with the aid of the brain mapping and monitoring techniques. ${ }^{2}$ Cortical mapping allowed us to determine the cortical safe entry zone. Subcortical mapping was performed to identify, from the beginning of resection, the functional boundaries surrounding the tumor. In the historical cohort (group B), only motor, visual, and language tracts were identified, whereas in the prospective cohort (group A) the standard procedure was performed with the mapping of the pathways belonging to praxis functions (HMt) (Table 3). Once the subcortical tracts were identified and the tumor was functionally disconnected, the mass was finally removed. During performance of the $\mathrm{HMt}$, low-frequency stimulation $(60 \mathrm{~Hz})$ was applied to cortical and subcortical structures by using the same current intensity delivered for language mapping $(2.75 \pm 0.93$ $\mathrm{mA})$.

\section{Hand Manipulation Task}

The device used for the HMt consists of a small cylindrical handle (diameter $2 \mathrm{~cm}$ and length $6 \mathrm{~cm}$ ) inserted inside a fixed rectangular base $(3 \times 3 \mathrm{~cm}$ and $9 \mathrm{~cm}$ long) driving a worm screw. The rectangular base was kept stable and close to the patient's hand along the armrest of the operating table, while the patient, with the thumb and index finger, grasped, held, rotated, and released the cylindrical handle. The cylindrical handle was kept close to the patient's hand, and he or she was allowed to perform the precision grip without a preceding reaching movement. The patient was asked to perform the task with a self-paced but regular rhythm. The task was performed in the absence of vision (haptically driven) to prevent head movement during the surgery. The lack of visual feedback was not expected to reduce the sensitivity of the test or to affect the behavioral outcome, because the parietofrontal circuits underlying praxis are active during visual as well as haptically guided movement.

We evaluated the intraoperative HMt performance during DES as follows. During execution, the patient's performance was monitored with visual inspection and online electromyographic (EMG) recording of the main hand and forearm muscles. At the beginning, patients performed 10 seconds of movement (without stimulation) to reach a stable task execution (baseline) assessed by monitoring both behavioral outcome and EMG activity. Then, randomly during task performance, the surgeon started the stimulation of the areas of investigation with a 3-to 4-second interval among different sites, to avoid dragging effects. A neuropsychologist reported to the surgeon the hand's behavioral outcome and/or the patient's somatic sensations. To increase the accuracy of the procedure, well-trained neuropsychologists, with experience acquired by a long training period and by exposure to a significant number of procedures, were charged with the evaluation of HMt performance. Moreover, during the procedures the task was often monitored online by 2 neuropsychologists, thus reducing the risk of subjective bias. The accurate presurgical training of the patients allowed the evaluators to be aware of the personal performance of each patient, thus enabling detection of intraoperative performance errors with high accuracy. Importantly, during brain mapping, the neuropsychologists were completely blinded to the site stimulated, the type of tumor, and the EOR, which excluded possible bias due to a priori anatomofunctional knowledge. Only the anatomical position of the tumor was available to the evaluator before surgery.

During the procedure, when the stimulation actually interfered with the task, an interval of 3-4 seconds preceded the next stimulation to allow the patient to regain a stable task execution. A stimulation site was deemed effective when it interfered with the task for at least 3 nonconsecutive trials. Patients with frontal tumors were positioned supine and semisitting (head of bed elevation $30^{\circ}$ ) with the head slightly tilted toward the contralateral side of the tumor. Patients with parietal tumors were positioned laterally, lying on the side contralateral to the lesion. In both surgical positions, the hand contralateral to the tumor was free to move and execute the task.

\section{Statistical Analysis}

The following analyses were performed to compare groups A and B: 1) the occurrence of postoperative neurological (visual, sensory, motor, language, and memory) deficits; 2) the occurrence of ideomotor apraxia and constructional abilities at the scheduled time point (postop- 
TABLE 3. Cortical and subcortical functional resection boundaries in the 2 groups

\begin{tabular}{|c|c|c|c|c|c|}
\hline \multirow[b]{2}{*}{ Hemisphere } & \multirow[b]{2}{*}{ Lobe } & \multicolumn{2}{|c|}{ Group A } & \multicolumn{2}{|c|}{ Group B } \\
\hline & & Cortical & Subcortical & Cortical & Subcortical \\
\hline \multirow[t]{5}{*}{ Dominant } & Frontal & M1 (motor responses) & M1 (motor responses) & M1 (motor responses) & M1 (motor responses) \\
\hline & & vIPM (speech \& HMt) & vIPM (speech \& HMt) & vIPM (speech) & vIPM (speech) \\
\hline & & & SMA/dPM (speech \& HMt) & & SMA/dPM (speech) \\
\hline & Parietal & S1-dorsal (HMt) & S1-dorsal (HMt) & SMG (language) & SMG (language) \\
\hline & & $\begin{array}{l}\text { SMG (HMt, language) } \\
\text { AG (language) }\end{array}$ & SMG (language, HMt) & AG (language) & \\
\hline \multirow[t]{4}{*}{ Nondominant } & Frontal & M1 (motor responses) & M1 (motor responses) & M1 (motor responses) & M1 (motor responses) \\
\hline & & vIPM (HMt) & SMA/dPM (HMt) & & \\
\hline & Parietal & S1-dorsal (HMt) & S1-dorsal (HMt) & M1 \& S1 (motor responses) & M1 \& S1 (motor responses) \\
\hline & & SMG (HMt) & SMG (HMt) & & \\
\hline
\end{tabular}

$\mathrm{dPM}=$ dorsal premotor area; SMA = supplementary motor area.

eratively and at $1-3$ months), globally and according to tumor location (parietal vs frontal) and the hemisphere affected (dominant vs nondominant); and 3) the EOR.

Analysis was performed with IBM SPSS statistical software (IBM Corp.) by using multiple chi-square tests (Pearson chi-square test) comparing the incidence of the different types of deficits between the 2 groups.

\section{Results}

\section{Functional Outcome}

In the postoperative period, the incidence of visual, motor, and other deficits (e.g., language) was not different in groups $\mathrm{A}$ and $\mathrm{B}$ (visual: $\mathrm{F}=3.385, \mathrm{p}=0.06$; motor: $\mathrm{F}=$ $0.007, p=0.936$; other: $F=1577, p=0.209$ ), whereas the incidence of postoperative somatosensory deficit was higher in controls (group B: $\mathrm{F}=10.053, \mathrm{p}=0.002$ ) (Fig. 2A).

As the main result, groups $\mathrm{A}$ and $\mathrm{B}$ differed significantly ( $p<0.001)$ in the incidence of ideomotor apraxia, irrespective of the hemisphere, both at 5 days (group A, 21/74 [28.4\%] vs group B, 27/38 [71.1\%]) and at 1-3 months (group A, 6/68 [8.8\%] vs group B, 18/38 [47.4\%]), with a higher prevalence of deficits in group B. Conversely, the incidence of constructional deficits was not statistically different at 5 days (group A, 35/66 [53\%] vs group B, $21 / 35$ [60\%]; $\mathrm{F}=0.450, \mathrm{p}=0.502$ ) and $1-3$ months (group $\mathrm{A}, 16 / 60$ [26.7\%] vs group B, 14/39 [35.9\%]; $\mathrm{F}=0.954, \mathrm{p}$ $=0.329)$ (Fig. 2B).

When the incidence of apraxia was analyzed according to the affected hemisphere, the following results were found.

1) For tumors located in the dominant hemisphere, the incidence of ideomotor apraxia was higher in group B, both at 5 days (group A, 17/51 [33.3\%] vs group B, 20/23 [87\%]; $\mathrm{F}=18.232, \mathrm{p}<0.001)$ and at $1-3$ months after surgery (group A, 6/49 [12.2\%] vs group B, 14/22 [63.6\%]; $\mathrm{F}=19.818, \mathrm{p}<0.001)$. Conversely, the incidence of constructional deficits showed no significant difference between groups at 5 days (group A, 25/45 [55.6\%] vs group $\mathrm{B}, 10 / 22[45.5 \%] ; \mathrm{F}=0.604, \mathrm{p}=0.437)$ and $1-3$ months (group A, 13/42 [31\%] vs group B, 6/21 [28.6\%]; F = 0.038, $\mathrm{p}=0.846)$ (Fig. 2C).

2) For tumors located in the nondominant hemisphere, the lower incidence of ideomotor apraxia in group A with respect to group $\mathrm{B}$ emerged in the long-term (group A, $0 / 19$ [0\%] vs group $\mathrm{B}, 4 / 16[25 \%] ; \mathrm{F}=5.363, \mathrm{p}=0.021$ ) rather than in the acute postoperative period (group A, $4 / 23$ [17.4\%] vs group B, 7/15 [46.7\%]; $\mathrm{F}=3.783, \mathrm{p}=$ 0.052). The incidence of constructional deficits, which was statistically different between groups at 5 days (group A, 10/21 [47.6\%] vs group B, 11/13 [84.6\%]; F = 0.604, p $=0.031$ ), was eventually superimposable in the long term (group A, 3/18 [16.7\%] vs group B, 8/18 [44.4\%]; F = 3.273, $\mathrm{p}=0.070)($ Fig. 2D).

The overall results demonstrate the efficacy of the HMt in decreasing the incidence of postoperative ideomotor apraxia. A descriptive analysis of the frequencies in patients belonging to group A showed that the overall incidence of apraxia was higher when the tumor affected the parietal versus the frontal lobe (acute apraxia: $38.5 \%$ vs 22.9\%; long-term apraxia: $16 \%$ vs $4.7 \%$ ). The same descriptive analysis showed that patients with tumor located in the nondominant hemisphere fully recovered within 1-3 months, whereas when the dominant hemisphere was affected the recovery within 3 months was not yet complete, which was consistent with the prominent role of the left hemisphere in ideomotor apraxia (Fig. 3). The majority of patients in group B (85.3\%) required postoperative motor rehabilitation, whereas only a minority $(3.8 \%)$ of those in group A required rehabilitation. A larger number of patients tested intraoperatively with the HMt is needed to statistically confirm the impact of the lobe and hemisphere affected in group A.

\section{Feasibility and Reproducibility in the Frontal and Parietal Lobes}

The HMt was feasible in all patients harboring parietal or frontal tumors (Fig. 4). In the frontal lobe, there were 2 main cortical responsive sites disrupting the regularity of movement: the vlPM ${ }^{11}$ and the hand knob of the primary motor cortex (M1). In both sites, DES disrupted task performance with different features: vlPM stimulation caused an almost complete arrest of movement, paralleled by an arrest of muscle activity, whereas M1 evoked an involuntary tetanic contraction of hand muscles. Stimulation of 
Hand manipulation (group $\mathrm{A}$ ) $\mathrm{n}=79$

A Control (group B) $n=41$
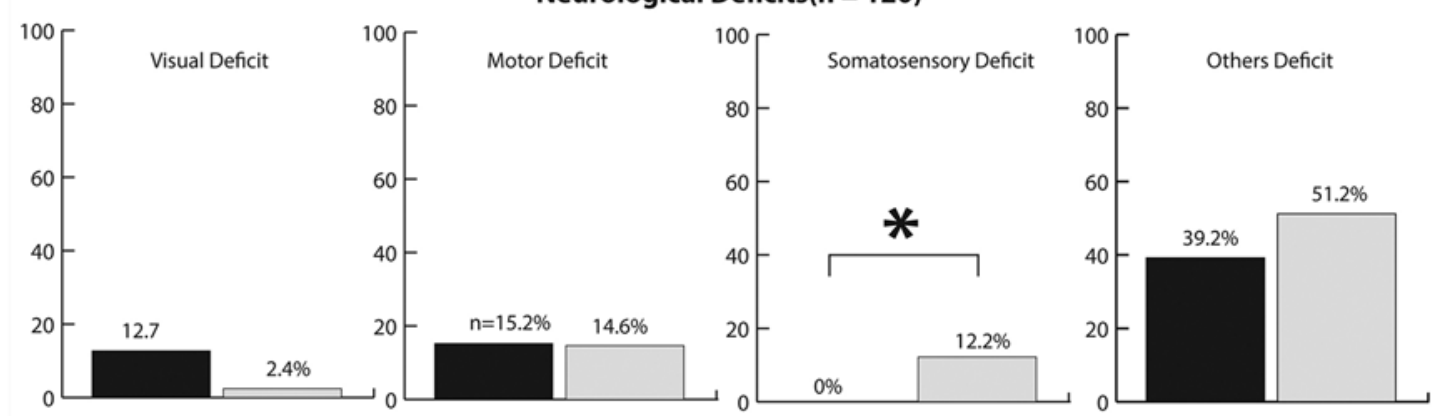

$\mathrm{B}$

Apraxia Deficits (population profile)

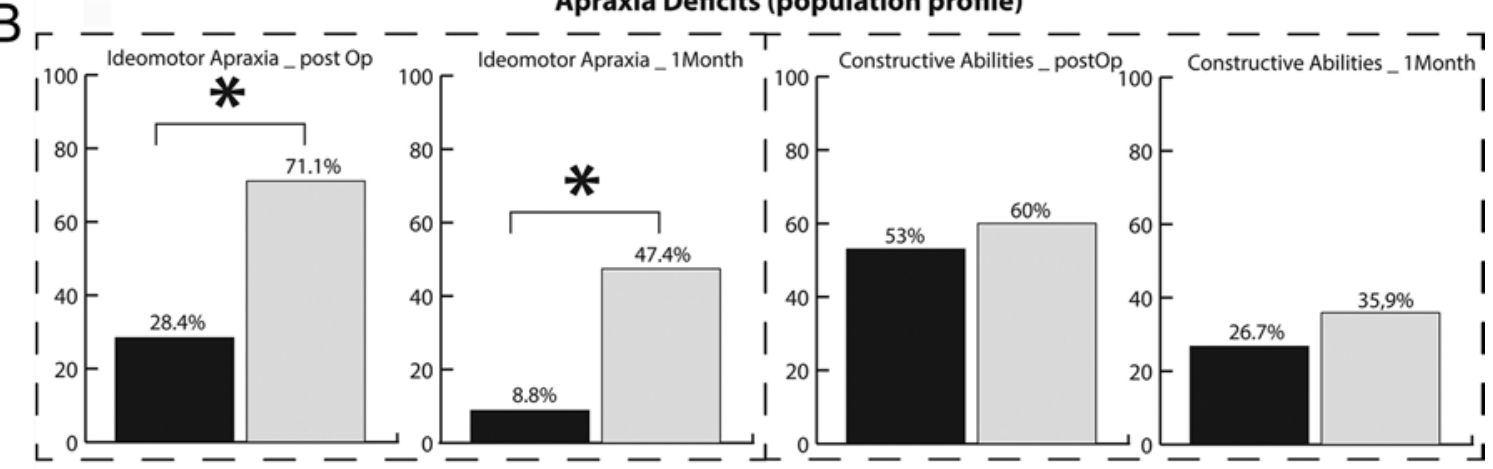

\section{Apraxia Deficits vs Hemisphere}
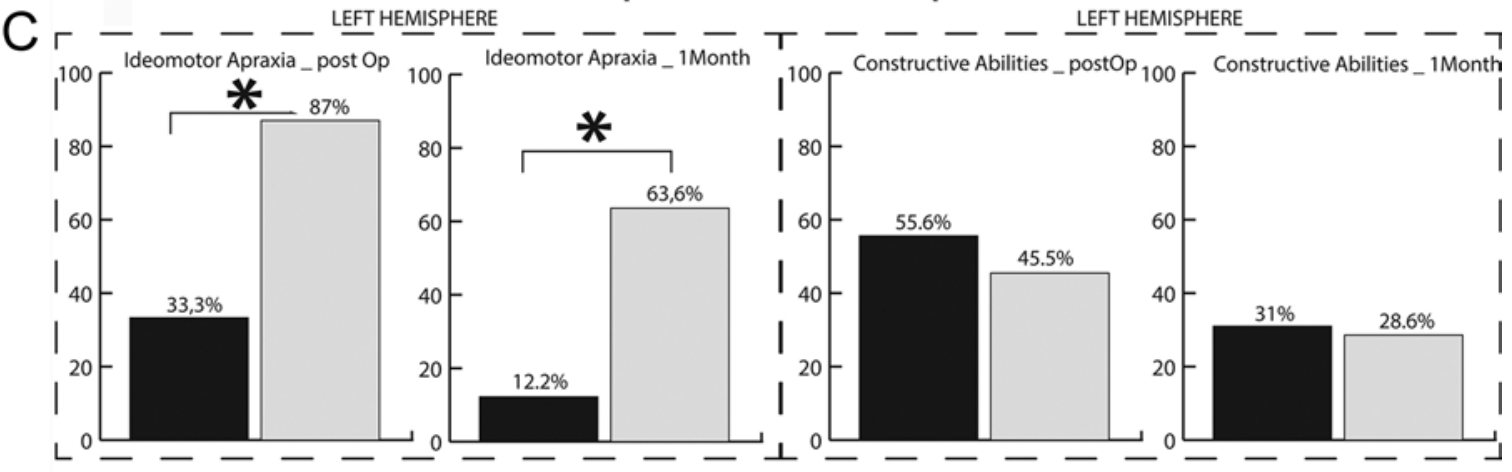

D $ᄃ---$ RIGHT HEMISPHERE
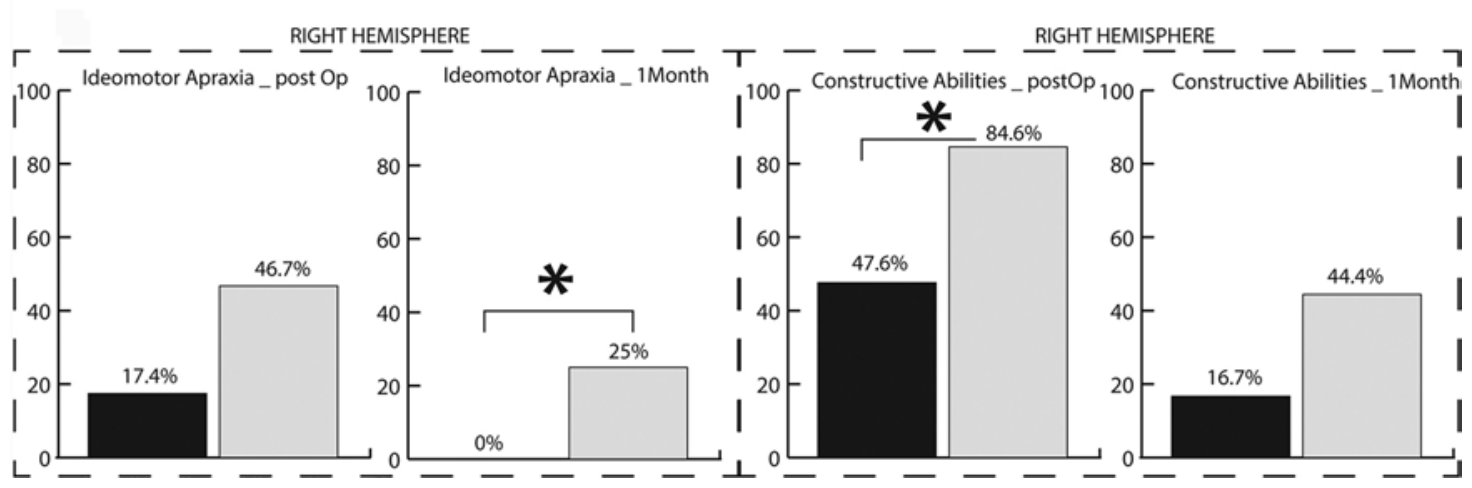

FIG. 2. Bar graphs showing postoperative clinical features assessed in patients belonging to group $A$ (HMt) and group $B$ (control). Asterisks indicate a statistically significant difference. A: The percentage distribution of the incidence of deficits observed at neurological examination 5 days after surgery. Visual = visual field deficits; motor = strength deficits; somatosensory = sensory deficits; others = language, memory, and comprehension deficits. B: The percentage distribution of the incidence of ideomotor or constructional apraxia, assessed at 5 days after surgery (postOp) and at 1 month in groups A and B. The ideomotor-apraxia test ${ }^{25}$ was used to assess the incidence of apraxia. $\mathbf{C}$ and $\mathbf{D}$ : The percentage distribution of the incidence of apraxia (ideomotor and constructional) measured in tumor patients at 5 days and 1 month after surgery in the 2 groups relative to the surgically treated hemisphere: dominant hemisphere (C) and nondominant hemisphere (D). 


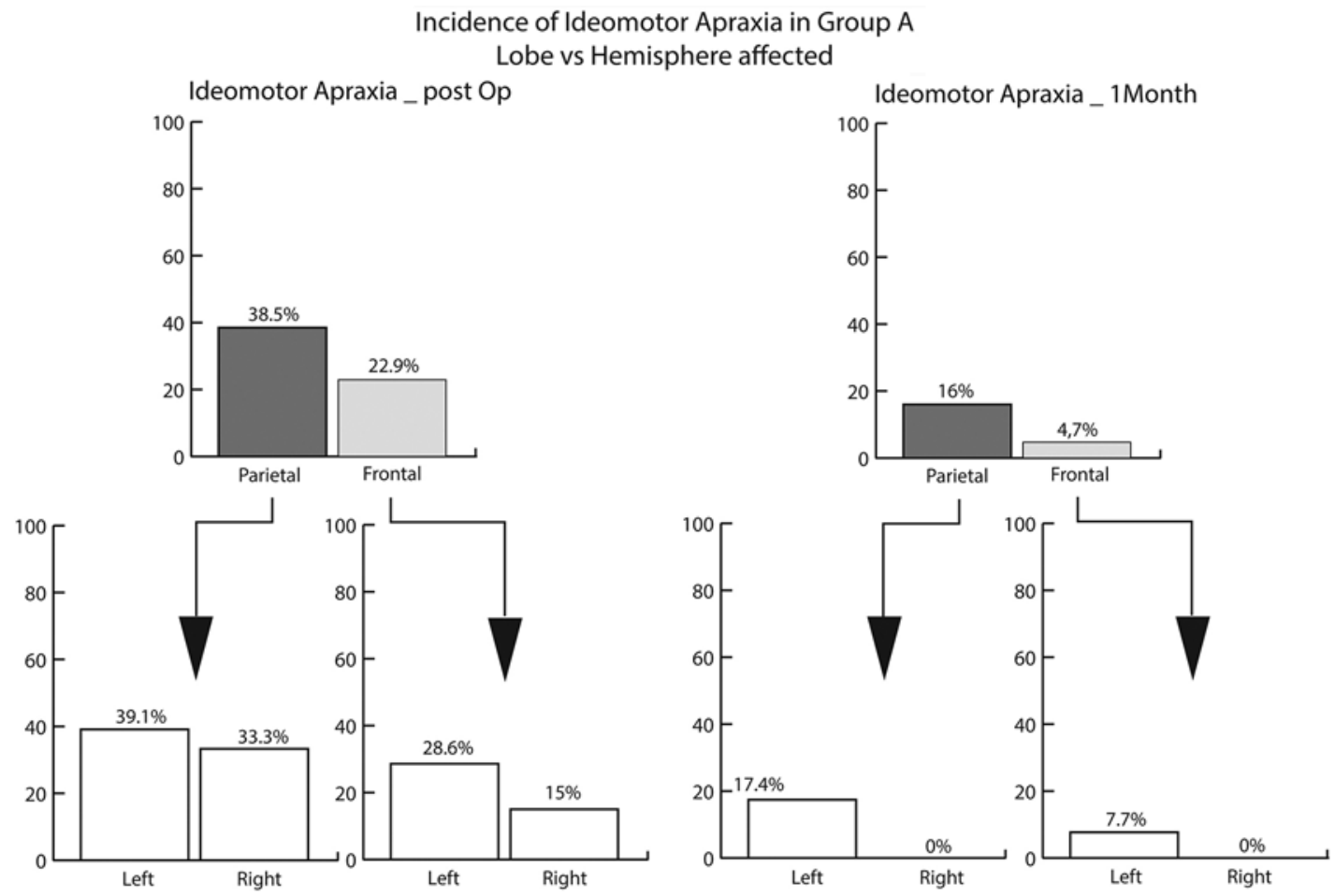

Incidence of Constructive Deficits in Group A

Lobe vs Hemisphere affected
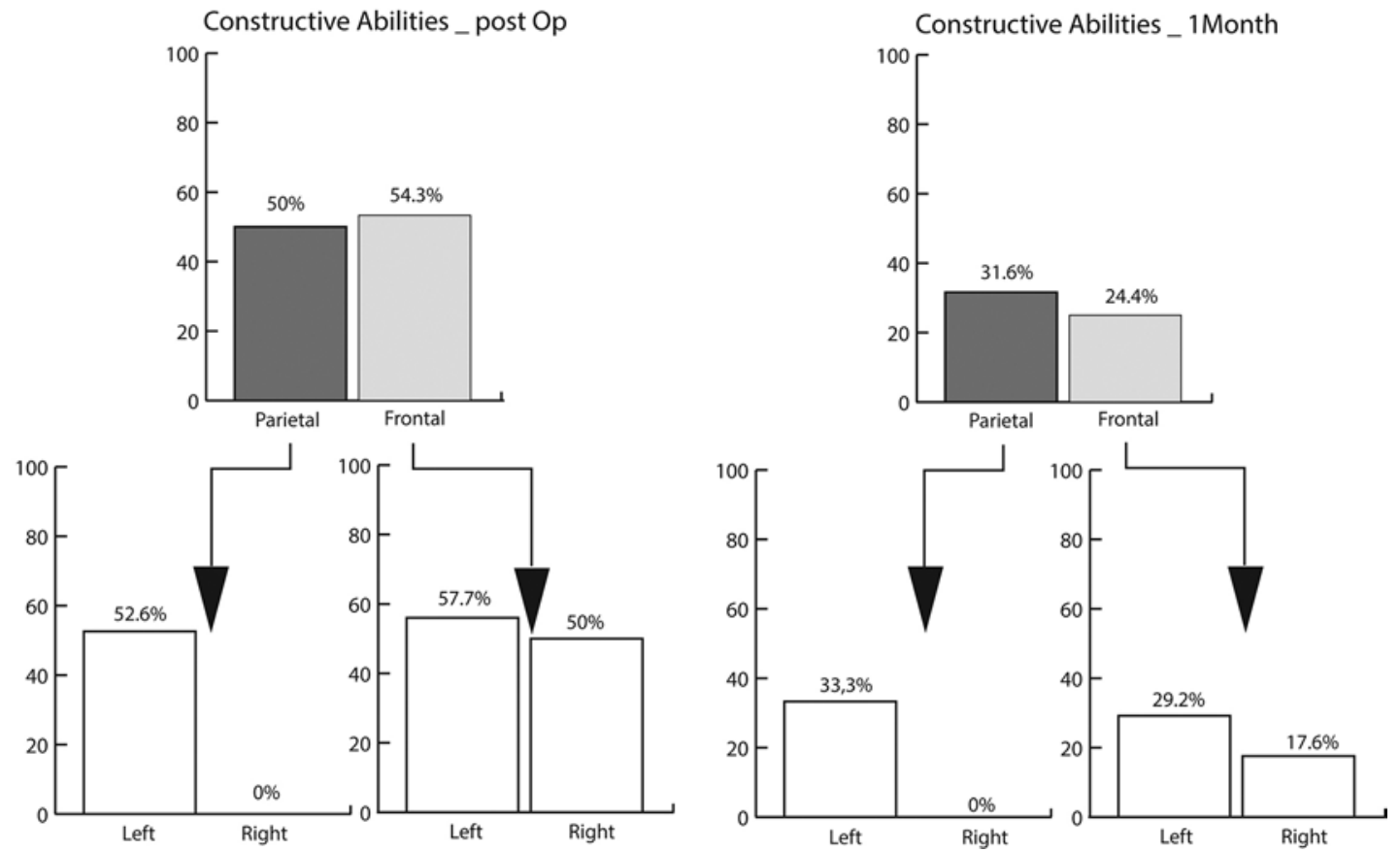

FIG. 3. Bar graphs showing the incidence of apraxia in patients belonging to group $A(H M t)$. The percentage distribution of incidence of ideomotor apraxia (upper panels) and constructional apraxia (lower panels) was qualitatively analyzed according to the treated lobe (parietal or frontal) and the affected hemisphere (left: dominant; right: nondominant).

the inferior, superior, and middle frontal gyri was ineffective. In the parietal lobe (Fig. 5), the responsive sites were the primary somatosensory cortex (S1) and the SMG; the former was associated with clonic twitches of the hand and fingers, and the latter showed similar features to the
vlPM block. Despite these similarities, the responsiveness of the vlPM to DES during the HMt was higher than that in the SMG (94\% vs 25\%) when stimulated with the same intensity. By increasing current intensity (average increase $1 \mathrm{~mA}$, range $0.5-1.5 \mathrm{~mA}$ ), the responsiveness increased to 

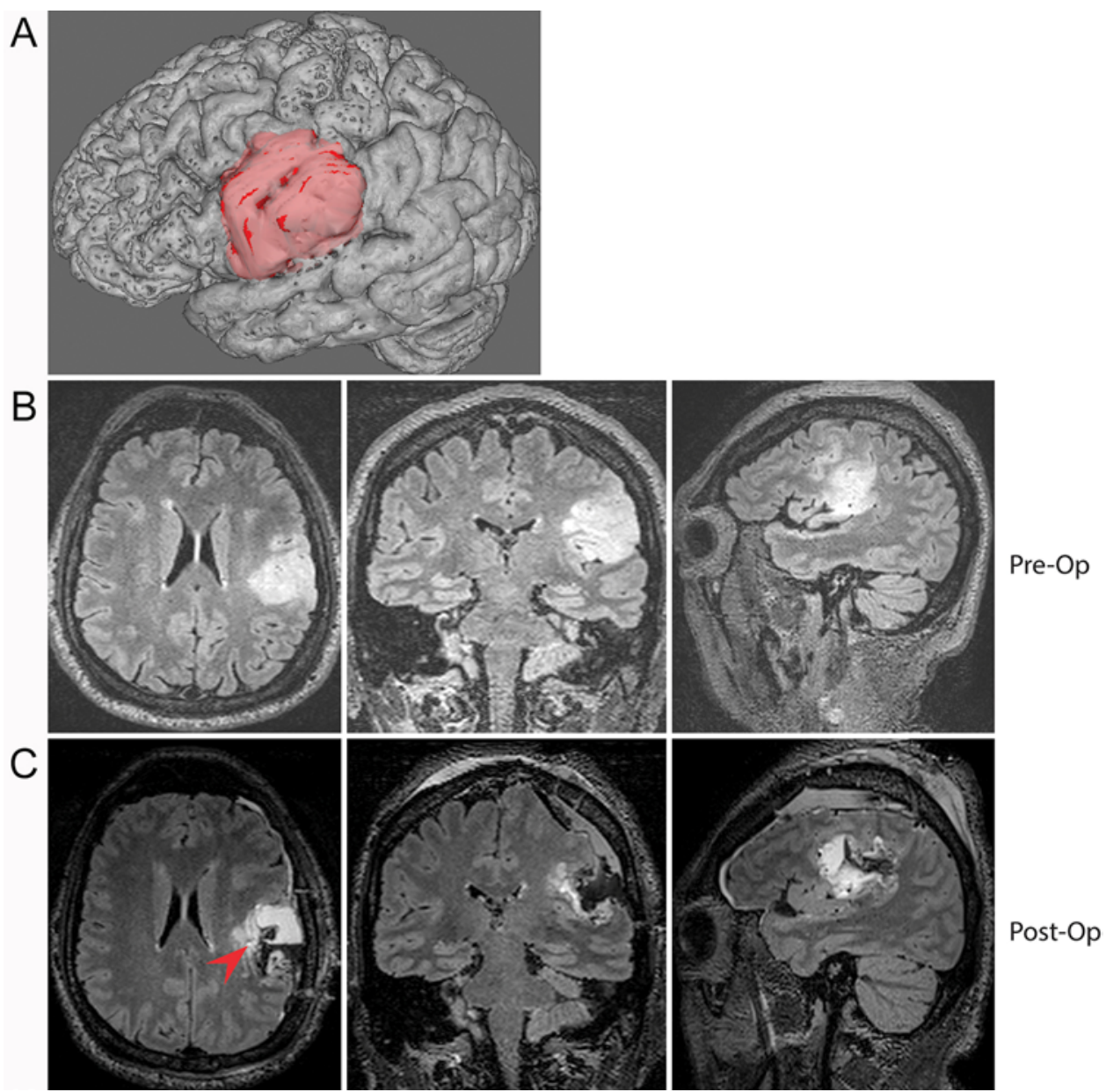

FIG. 4. Representative case of a patient with a glioma (grade II IDH1 mutated, codeleted, ATRX intact oligodendroglioma) localized in the dominant frontoparietal areas subserving both language and praxis functions. A: The tumor volume manually segmented is shaded in red. B and C: Representative preoperative (B) and postoperative (C) FLAIR (axial, coronal, and sagittal) images. A limited tumor remnant (visible in the postoperative axial image) was located proximal to the site where phonemic paraphasias were induced by subcortical mapping (red arrowhead). Figure is available in color online only.

$75 \%$. Subcortically, in the frontal lobe, interferences were induced below the vlPM and the dorsomesial sector directly anterior to the precentral sulcus. In the parietal lobe, interferences were induced below the postcentral gyrus and between the SMG and the fundus of the ventral postcentral gyrus. Here the surgeon very clearly distinguished, during mapping, the fibers associated with HMt interference, which were discrete and separate from the M1 descending fibers and language-associated tracts (Table 3). Cortical and subcortical HMts increase the mapping time by 6 minutes on average.

\section{Oncological Results}

The EOR was calculated by the manual segmentation method in both groups without showing statistical differences $(p=0.183$; group A mean EOR $96.37 \%$, group B mean EOR 93.3\%).

\section{Discussion}

The brain mapping technique is crucial for brain tumor resection in that it allows surgeons to extend the resection while preserving the functional integrity of their patients. Standard brain mapping techniques are highly efficient for the identification of motor sites in patients with tumors in descending motor pathways. However, careful scrutiny of patients with tumors located around the pre- and postcentral gyrus and infiltrating the subcortical parietofrontal fibers who were treated by our team between 2013 and 2014 with the standard intraoperative neurophysiological approach revealed the occurrence of apraxia in $30 \%$ of cases despite the absence of motor (i.e., palsy, tremor, ataxia, or hypokinesia and/or hyperkinesia) and somatosensory deficits (i.e., deafness, blindness, or visual and tactile agnosia).

Apraxia is frequently underestimated in neurosurgical patients, and a comprehensive assessment of its incidence after surgery for brain tumor removal is lacking. Apraxia is a relevant disability that significantly impairs daily human activities requiring skilled hand abilities, such as the use of tools to achieve a desired goal. To prevent this deficit, the neural structures controlling hand praxis functions must be preserved intraoperatively. Unfortunately, a specific hand motor task to be used for this purpose in this context is lacking. An attempt by patients to perform a 

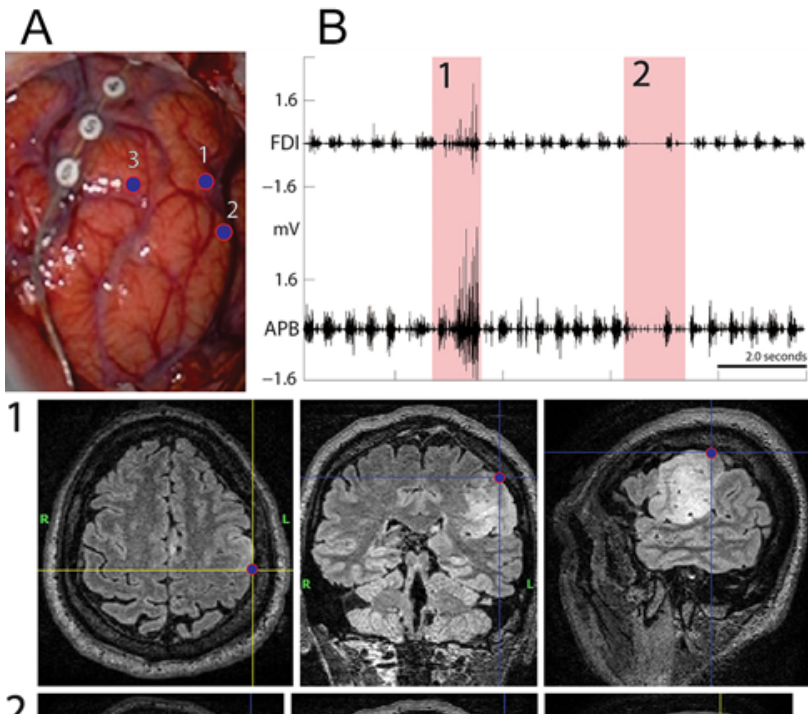

2
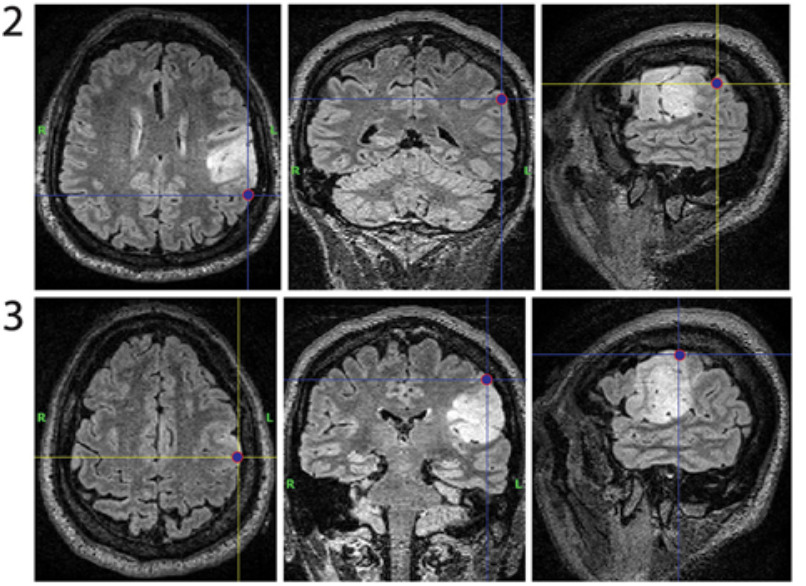
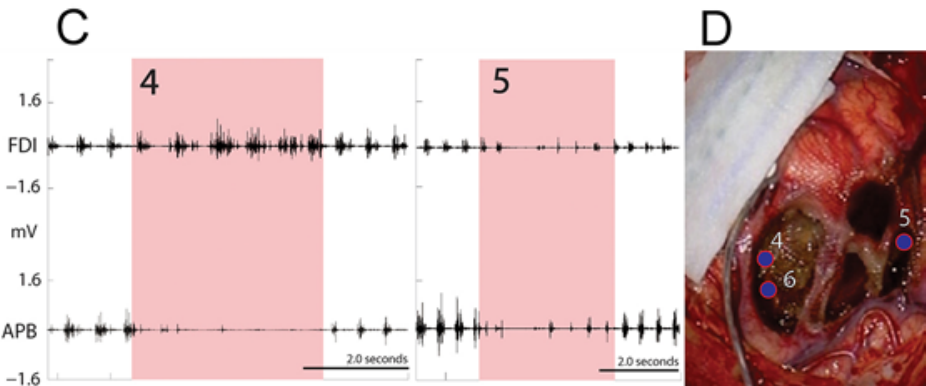

4
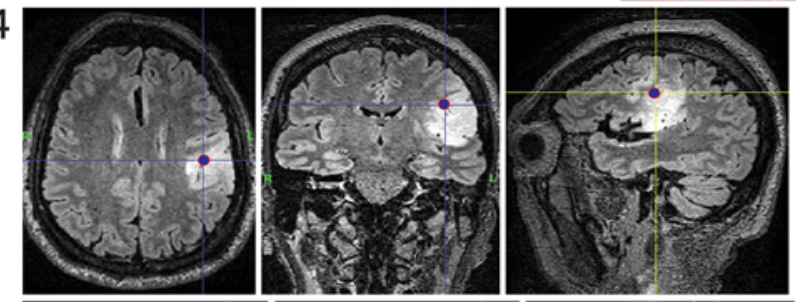

5
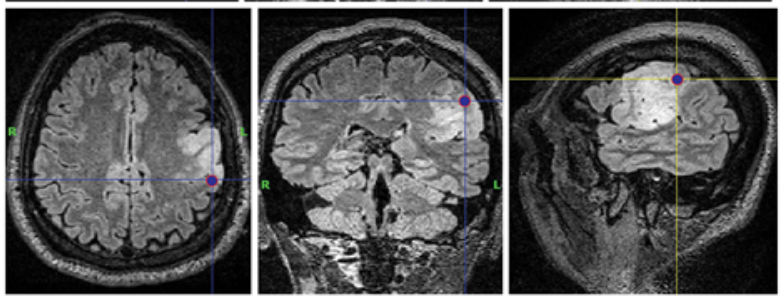

6
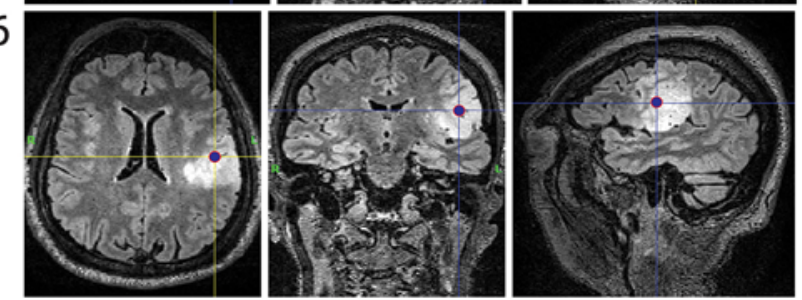

FIG. 5. Representative case of a glioma (reported in Fig. 4) showing cortical and subcortical sites in which DES evoked interference during the language task and the HMt. Left panels: Recorded sites of cortical stimulation. A: Intraoperative photograph of the area under the surgical flap. The locations of the sites in which DES induced interference are designated by blue circles. Sites 1 (S1) and 2 (SMG) were associated with disruption of the HMt; in site 3 (vIPM), DES induced speech dysarthria. The cortical strip was placed over M1. B: The pattern of EMG muscle activation recorded in 2 muscles (first dorsal interosseous [FDI] and abductor pollicis brevis [APB]) during the performance of the HMt. The pattern appears as a burst contraction; in site 1 the interference induced by DES on S1 (clonic twitches) is shown, and in site 2 the interference induced by DES on SMG (abolishment of FDI and APB activity) is shown. The time window corresponding to stimulation is indicated by the pink area. 1-3: The location of each site on representative axial, coronal, and sagittal FLAIR images (registered with neuronavigation). Right panels: Recorded sites of subcortical stimulation. C: The pattern of interference on the EMG signal recorded in FDI and APB during stimulation in sites 4 and 5. The stimulation at site 4 abolished APB activity, leaving the FDI activation; HMt interference at site 5 resembles the effect obtained by cortical stimulation of SMG. The time window corresponding to stimulation is indicated by the pink area. D: Intraoperative photograph of the area under the surgical flap at the end of resection. A patty covers the cortical strip. Blue circles 4 and 5 indicate the stimulation sites associated with disruption of the HMt. In site 6, below and close to site 4, DES induced phonemic paraphasia. 4-6: The location of the sites on representative axial, coronal, and sagittal FLAIR images (registered with neuronavigation). Sites 4 and 6 are located at the anterior margin of the surgical cavity, and site 5 at the superior margin. Figure is available in color online only.

repetitive flexion-extension of the contralateral arm during awake surgery has been reported in a few cases. ${ }^{24}$ This was done without a hand-object interaction as a fundamental requirement to study the hand movements devoted to object prehension and manipulation., ${ }^{4,22}$

To overcome these limitations, we developed a new intraoperative task, the HMt, resembling a hand-object ecological interaction and requiring, to be executed successfully, the integrity of the frontoparietal praxis network.,12 The HMt is proposed as a mapping tool during surgery, based on the hypothesis that DES applied on praxis-eloquent cortical and/or subcortical sites is expected to disrupt the ongoing HMt. At present, no specific task has been used during the brain mapping technique to avoid apraxia. The standard neuropsychological apraxia assessment cannot be applied during intraoperative awake surgery, which requires, due to clinical constraints, an adequate task that is easily performed by patients and at the same time has a clear behavioral output to be monitored by the neurophysiologist. The reliability of the HMt de- 
signed for the intraoperative setting deserves comment. To be correctly performed, the HMt requires complex finger movements usually involved in the most dexterous handobject interaction, i.e., the precision grip, probably resembling the motor task reported in the literature to investigate, in both monkeys and humans, the neural control of grasping and manipulation., ${ }^{4,22}$ Moreover, the HMt, which requires a higher dexterity with respect to a side or whole hand grip, seems adequate to preserve both the more and less highly skilled grip. Notably, the HMt is specifically designed for the evaluation of praxis skills, given that it simulates the actual use of a tool rather than an aimless hand-object interaction. Moreover, the HMt is perfomed in the absence of an external artificial cue and therefore is completely based on ideational self-generated programming, and both of these features are crucial aspects for the evaluation of apraxia.

The effect of DES was evaluated in terms of behavioral outcome and EMG activation of the most involved hand muscles and was reported in real time to the surgeon. To assess the intraoperative efficacy of the HMt to unveil and preserve the praxis network, we investigated the occurrence of postoperative ideomotor apraxia in patients undergoing mapping with and without the HMt. The introduction of the HMt dramatically decreased the incidence of immediate and long-term postoperative apraxia. Moreover, the comparison of praxis and constructional abilities reveals that the HMt seems more selective for ideomotor apraxia than for constructional abilities, except in patients in whom the nondominant hemisphere is affected, and in whom the HMt equally reduced the occurrence of both deficits (Fig. 2D). Accordingly, the clinical recommendation is to apply the task (in awake patients) independently according to hemispheric dominance. However, despite the clear effect of the HMt on ideomotor apraxia, there was a residual percentage of patients with deficits (8.8\%), which mainly involved the parietal lobe in the dominant hemisphere rather than the frontal lobe (Fig. 3). This finding suggests that the task is highly efficient in the frontal lobe in identifying the anterior components of the parietofrontal network running below the vlPM. Concerning the parietal lobe, the data highlight possible limitations of the HMt in detecting, with the same efficacy shown for the frontal lobe, the posterior component of the praxis network. Because the parietal lobe is mainly devoted to the integration of multiple inputs of sensory information during action, in the future the $\mathrm{HMt}$ needs to be optimized to assess these functional features, possibly also introducing-with haptic control exercised by the patient-visual information regarding the ongoing hand-object interaction. Our hypothesis is that the parietal lobe (particularly the SMG) as a multisensory associative area might require the simultaneous influx of multiple sensory inputs to become active, as possibly the haptic inputs generated during HMt performance only partially activate the SMG.

Consistent with this hypothesis, in our sample of patients the SMG required a higher intensity than the M1, vlPM, and S1 to evoke an interference of the ongoing HMt. This higher intensity was probably necessary to force the artificial activation of the SMG. However, we cannot exclude the possibility that this intensity-related effect might also be due to the peculiar cytoarchitectonic features of the SMG, which are different from those of the other areas and lead to a lower excitability.

We can conclude that the HMt is very feasible; it is easily performed by all patients, independently of patient age and tumor location. From the surgical point of view, the emergence of specific patterns of interference on task execution (both behavioral and EMG) was associated with the different parietofrontal eloquent sites, providing surgeons with an invaluable tool, similar to a fingerprint, to navigate within the praxis network during resection: the M1 block is characterized by tonic muscle activation, the $\mathrm{S} 1$ by clonic twitches and release of the object, and the SMG and vlPM by the arrest of movement without muscle activation. Moreover, the stimulation of the dorsomesial sectors of the premotor cortex induced a slow deceleration of the movement and a loss of rhythmicity in the hand-object interaction. Interestingly, when stimulated in the resting state condition (patient motionless), the S1, SMG, and vlPM failed to elicit motor responses, at most inducing weak somatosensory sensations in the hand and forearm. Similar features were observed when DES was applied to subcortical sites below the areas described. Interestingly, when the eloquent subcortical parietal and frontal sites were stimulated during the resting state with high-frequency stimulation (a stimulation paradigm highly efficient in mapping the corticofugal components [corticospinal tract] of the motor areas), ${ }^{2}$ no motor responses were evoked, a result that suggests an anatomofunctional decoupling between the praxis network and the corticospinal tract.

The preservation of the parietofrontal circuit underlying praxis abilities was achieved while keeping the optimal surgical and oncological end point (an EOR that was not affected by the introduction of the HMt). The higher occurrence of ideomotor apraxia in the acute postoperative period confirmed that the functional boundaries were indeed reached, as reported for other functions in the awake setting. The HMt and the associated subcortical fibers were closely intermingled with those inducing language or motor responses, but always functionally and discretely separated from them. This separation provides a reasonable explanation for why the functional identification enabled by the HMt did not result in any significant change in the EOR but had a strong positive impact on outcomes associated with functional hand dexterity.

\section{Conclusions}

The HMt is a safe, feasible, and efficient tool for intraoperative identification of the hand praxis network, a fundamental requisite to prevent long-term ideomotor hand apraxia independently of age, side, or hemispheric dominance. Further studies are needed to improve the intraoperative identification of the neural structures underlying the visuomotor abilities, as well as to avoid long-term constructional deficits.

\section{References}

1. Basso A, Capitani E, Laiacona M: Raven's coloured progressive matrices: normative values on 305 adult normal controls. Funct Neurol 2:189-194, 1987 
2. Bello L, Riva M, Fava E, Ferpozzi V, Castellano A, Raneri F, et al: Tailoring neurophysiological strategies with clinical context enhances resection and safety and expands indications in gliomas involving motor pathways. Neuro Oncol 16:1110-1128, 2014

3. Binkofski F, Buccino G, Posse S, Seitz RJ, Rizzolatti G, Freund H: A fronto-parietal circuit for object manipulation in man: evidence from an fMRI-study. Eur J Neurosci 11:3276-3286, 1999

4. Borra E, Gerbella M, Rozzi S, Luppino G: The macaque lateral grasping network: a neural substrate for generating purposeful hand actions. Neurosci Biobehav Rev 75:65-90, 2017

5. Caffarra P, Vezzadini G, Dieci F, Zonato F, Venneri A: ReyOsterrieth complex figure: normative values in an Italian population sample. Neurol Sci 22:443-447, 2002

6. Caffarra P, Vezzadini G, Dieci F, Zonato F, Venneri A: Una versione abbreviata del test di Stroop: dati normativi nella popolazione italiana. Nuova Riv Neurol 12:111-115, 2002

7. Carlesimo GA, Caltagirone C, Gainotti G, Fadda L, Gallassi R, Lorusso S, et al: The Mental Deterioration Battery: normative data, diagnostic reliability and qualitative analyses of cognitive impairment. Eur Neurol 36:378-384, 1996

8. Crepaldi D, Aggujaro S, Arduino LS, Zonca G, Ghirardi G, Inzaghi MG, et al: Noun-verb dissociation in aphasia: the role of imageability and functional locus of the lesion. Neuropsychologia 44:73-89, 2006

9. De Renzi E, Faglioni P: Normative data and screening power of a shortened version of the Token Test. Cortex 14:41-49, 1978

10. Dovern A, Fink GR, Weiss PH: Diagnosis and treatment of upper limb apraxia. J Neurol 259:1269-1283, 2012

11. Fogassi L, Gallese V, Buccino G, Craighero L, Fadiga L, Rizzolatti G: Cortical mechanism for the visual guidance of hand grasping movements in the monkey: a reversible inactivation study. Brain 124:571-586, 2001

12. Fornia L, Ferpozzi V, Montagna M, Rossi M, Riva M, Pessina F, et al: Functional characterization of the left ventrolateral premotor cortex in humans: a direct electrophysiological approach. Cereb Cortex [epub ahead of print], 2016

13. Frey SH: Tool use, communicative gesture and cerebral asymmetries in the modern human brain. Philos Trans $\mathbf{R}$ Soc Lond B Biol Sci 363:1951-1957, 2008

14. Geschwind N: The apraxias: neural mechanisms of disorders of learned movement. Am Sci 63:188-195, 1975

15. Giovagnoli AR, Del Pesce M, Mascheroni S, Simoncelli M, Laiacona M, Capitani E: Trail making test: normative values from 287 normal adult controls. Ital J Neurol Sci 17:305309, 1996

16. Goldenberg G: Apraxia and the parietal lobes. Neuropsychologia 47:1449-1459, 2009

17. Hanna-Pladdy B, Heilman KM, Foundas AL: Ecological implications of ideomotor apraxia: evidence from physical activities of daily living. Neurology 60:487-490, 2003

18. Heilman KM, Gonzalez Rothi LJ: Apraxia, in Heilman KM,
Valenstein E (eds): Clinical Neuropsychology. New York: Oxford University Press, 1993, pp 141-163

19. Koski L, Iacoboni M, Mazziotta JC: Deconstructing apraxia: understanding disorders of intentional movement after stroke. Curr Opin Neurol 15:71-77, 2002

20. Laiacona M, Inzaghi MG, De Tanti A, Capitani E: Wisconsin card sorting test: a new global score, with Italian norms, and its relationship with the Weigl sorting test. Neurol Sci 21:279-291, 2000

21. Monaco M, Costa A, Caltagirone C, Carlesimo GA: Erratum to: Forward and backward span for verbal and visuo-spatial data: standardization and normative data from an Italian adult population. Neurol Sci 36:345-347, 2015

22. Nelissen K, Vanduffel W: Grasping-related functional magnetic resonance imaging brain responses in the macaque monkey. J Neurosci 31:8220-8229, 2011

23. Novelli G, Papagno C, Capitani E, Laiacona M, Vallar G, Cappa SF: Tre test clinici di ricerca e produzione lessicale. Taratura su soggetti normali. Arch Psicol Neurol Psich 47:477-506, 1986

24. Rech F, Herbet G, Moritz-Gasser S, Duffau H: Somatotopic organization of the white matter tracts underpinning motor control in humans: an electrical stimulation study. Brain Struct Funct 221:3743-3753, 2016

25. Spinnler H, Tognoni G: [Italian standardization of neuropsychological tests.] Ital J Neurol Sci Suppl 8:1-120, 1987 (Italian)

\section{Disclosures}

The authors report no conflict of interest concerning the materials or methods used in this study or the findings specified in this paper.

\section{Author Contributions}

Conception and design: Fornia, Bello. Acquisition of data: Fornia, Puglisi, Leonetti, Zuccon, Fava, Milani, Casarotti. Analysis and interpretation of data: Rossi, Fornia, Leonetti, Bello. Drafting the article: Rossi, Fornia. Critically revising the article: Rossi, Cerri, Bello. Reviewed submitted version of manuscript: Rossi, Bello. Approved the final version of the manuscript on behalf of all authors: Rossi. Statistical analysis: Fornia, Puglisi. Study supervision: Riva, Pessina, Cerri.

\section{Supplemental Information}

\section{Previous Presentations}

Some preliminary results of the study were given as an oral presentation at the European Association of Neurosurgical Societies (EANS) meeting in 2016 in Athens, Greece.

\section{Correspondence}

Marco Rossi: Università degli Studi di Milano, Italy. marco. rossi2@unimi.it. 